\title{
An experimental demonstration of resonant sideband extraction for laser-interferometric gravitational wave detectors
}

\author{
G. Heinzel ${ }^{1}$, J. Mizuno ${ }^{2}$, R. Schilling, W. Winkler, A. Rüdiger, K. Danzmann ${ }^{3}$ \\ Max-Planck-Institut für Quantenoptik, Hans-Kopfermann-Strasse 1, D-85748 Garching, Germany \\ Received 29 February 1996; accepted for publication 30 April 1996 \\ Communicated by P.R. Holland
}

\begin{abstract}
Resonant sideband extraction is a new optical configuration for laser-interferometric gravitational wave detectors with Fabry-Perot cavities in the arms. It reduces the thermal load on the beam splitter and the coupling mirrors of the cavities and also allows one to adapt the frequency response of the detector to a variety of requirements. We report the first experimental demonstration using a table-top setup at a detuned operating point. An increase of sensitivity by $6 \mathrm{~dB}$ was observed for artificial signals at frequencies above the arm cavity bandwidth, and the overall transfer function agreed well with theoretical predictions.
\end{abstract}

PACS: $04.80 . \mathrm{Nn} ; 07.60 . \mathrm{Ly} ; 42.25 . \mathrm{Hz} ; 95.55 . \mathrm{Ym}$

Keywords: Resonant sideband extraction; Michelson interferometer; Fabry-Perot; Mach-Zehnder; Gravitational wave detector;

Three-mirror cavity

\section{Introduction}

Direct detection of gravitational waves by laser interferometry seems possible within the next several years. Construction work has begun for several large detectors (e.g. LIGO in the U.S. [1], VIRGO [2] and GEO600 [3] in Europe, and others). One fundamental limit for the sensitivity of any optical detector is given by the photon counting error (shot noise). In order to achieve the desired high sensitivity, various extensions to the basic Michelson interferometer have been proposed, such as delay lines or Fabry-Perot cavities in the arms, power- and signal-recycling [4,5]. It can be shown [6] that for any of these configurations the shot-noise limited sensitivity (expressed as linear spectral density $\tilde{h}$ of strain in space producing the same output signal as the intrinsic detector noise) is proportional to $\sqrt{\Delta f_{\mathrm{BW}} / E}$, where $E$ is the amount of light

\footnotetext{
${ }^{1}$ E-mail: ghh@mpq.mpg.de.

${ }^{2}$ E-mail: mizuno@mpq.mpg.de.

${ }^{3}$ Also at Universität Hannover, Institut für Atom- und Molekülphysik, Appelstrasse 2, D-30167 Hannover, Germany.
} 


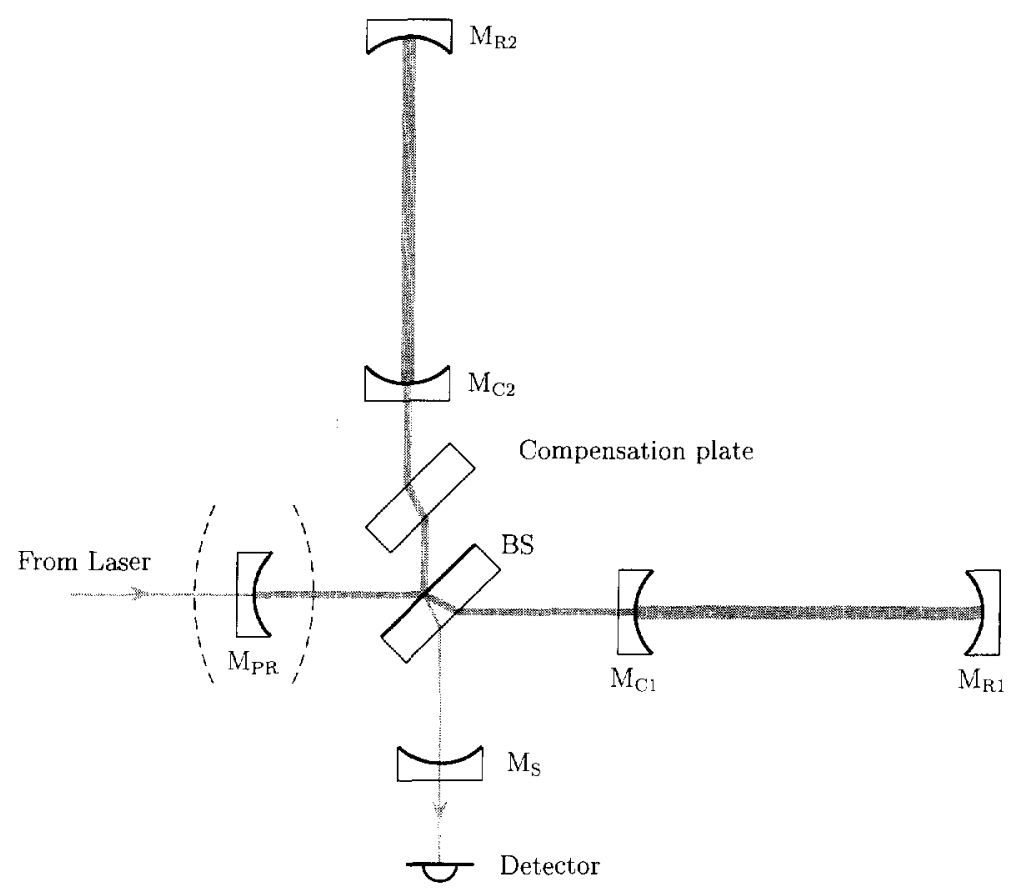

Fig. 1. Optical layout for resonant sideband extraction. Power recycling (with mirror $\mathrm{M}_{\mathrm{PR}}$ ) is an option not affecting the principle of RSE. The compensation plate may be included for reasons of symmetry between the two arms.

energy stored in the arms, and $\Delta f_{\mathrm{BW}}$ the detector bandwidth. All of the above mentioned schemes amount to increasing $E$ for a given physical arm length and laser power and/or modifying the detector bandwidth.

Among these schemes, power recycling is considered indispensable for all of the planned large detectors. The power gain achievable will, however, be limited by imperfect contrast as well as losses in the substrates of the beam splitter (and those of the arm cavity coupling mirrors). Furthermore, the high-power beam may cause absorption-induced thermal lensing and birefringence in the substrates [7-9].

By using high-finesse Fabry-Perot arm cavities, the high-power beam is confined to within the arm cavities and is not transmitted through any substrate. The usable finesse of the arm cavities was, however, believed to be limited by the required detector bandwidth.

Resonant sideband extraction (RSE) $[6,10]$ was proposed as a new optical configuration to overcome this limitation. In particular, the detector bandwidth can be made broader than the arm-cavity bandwidth, thereby permitting the use of high-finesse cavities in the arms. There exist non-detuned and detuned modes of operation, where "tuned" in this paper refers to making the signal extraction cavity resonant for the carrier, which yields the broadest possible detector bandwidth. The "detuned" modes permit a sensitivity peak to be obtained at an adjustable frequency.

The outline of this paper is as follows: In Section 2, we describe the frequency response of RSE, closely following Ref. [6]. Our experimental setup is described in Section 3. The results are discussed in Section 4.

\section{Frequency response of resonant sideband extraction}

Fig. 1 shows the basic optical layout for RSE. It resembles that of signal recycling with Fabry-Perot cavities in the arms, but here a different sub-wavelength positioning of the signal extraction mirror ( $\mathrm{M}_{\mathrm{S}}$ in Fig. 1) 


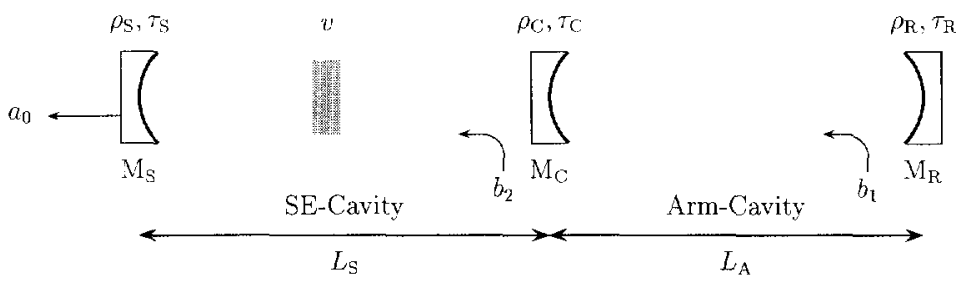

Fig. 2. Three-mirror cavity storing the signal sidebands.

produces a different effect, as described below.

To simplify the analysis, we assume all interfering beams to have the same polarization and transverse mode structure. To compute the frequency response, we also assume a 50/50 beam splitter, both arm cavities to be identical and in resonance with the incoming light of angular frequency $\omega_{0}$ (the "carrier"). Furthermore, we assume the interferometer to be operated at a dark fringe (at the detector port), i.e. all carrier light returning from the arms is interfering constructively towards the laser (or power-recycling mirror). Note that the amplitude reflectivity of the Michelson interferometer as a whole seen from either port is identical (if the beam splitter's transmittance equals its reflectivity), whatever the arms are.

A suitably oriented and polarized gravitational wave produces a phase modulation of opposite sign in the two arms. In the following we consider only a single frequency component of angular frequency $\omega_{\mathrm{g}}$. The phase modulation sidebands at $\omega_{0} \pm \omega_{\mathrm{g}}$ interfere constructively towards the signal extraction mirror $\mathrm{M}_{\mathrm{S}}$. After being reflected by $M_{S}$, they re-enter the interferometer, which in turn reflects them back to $M_{S}$. The signal sidebands are thus stored in a "split" cavity [11] composed of the Michelson interferometer and $\mathbf{M}_{\mathbf{S}}$. Under the above assumptions, this split cavity is equivalent to a three-mirror cavity (Fig. 2) formed by $\mathbf{M}_{S}, \mathrm{M}_{\mathrm{C}}$ and $\mathrm{M}_{\mathrm{R}}$, where the two identical arm cavities have been "folded" together to form a single cavity consisting of $M_{C}$ and $M_{R}$. (The notations in Fig. 2 are explained in the context of Eqs. (1) and (2)).

One possible interpretation of this three-mirror cavity is to consider the cavity as being composed of $\mathbf{M}_{\mathrm{R}}$ and a compound mirror formed by $\mathrm{M}_{\mathrm{S}}$ and $\mathrm{M}_{\mathrm{C}}$. The compound mirror (which we also call the "signal extraction cavity") has frequency-dependent transmittance and reflectivity. In signal recycling, this cavity is tuned to "anti-resonance" (i.e. centered between two successive resonances) so as to obtain a lower transmittance than that of $\mathrm{M}_{\mathrm{C}}$ alone, resulting in an increased storage time for the signal.

The purpose of RSE, on the other hand, is to reduce the storage time for the signal, in order to allow long storage times for the carrier without sacrificing detector bandwidth. This can be achieved by tuning the signal extraction cavity to resonance so that its transmittance for the signal frequencies of interest is higher than that of $\mathbf{M}_{\mathrm{C}}$ alone. For these frequencies the storage time in the three-mirror cavity is shorter than that in the unmodified arm cavity. In the non-detuned case, this reduction of the storage time results in an increased bandwidth.

To compute the frequency response we consider the light amplitudes going to and from each mirror in the scalar model (see Fig. 2). As input to the cavity we have $b_{1}$, which represents the signal sidebands produced by differential phase modulation of the carrier (e.g. by gravitational waves) in the arms. Of importance in our experiment is also a further input $b_{2}$, which corresponds to phase modulation sidebands produced between the beam splitter and arm cavity. Note that the carrier (in an ideal dark-fringe operation) never reaches $\mathrm{M}_{\mathbf{S}}$ and therefore is not considered in this picture.

The amplitudes at the different points are coupled by a set of linear equations. Solving this set for the output amplitude $a_{0}$ yields the transfer functions

$$
G_{1}(\omega, \delta)=\frac{a_{0}}{b_{1}}=\frac{\tau_{\mathrm{C}} \tau_{\mathrm{S}} v \mathrm{e}^{\mathrm{i} \omega\left(T_{\mathrm{A}}+T_{\mathrm{S}}\right)}}{1-\rho_{\mathrm{R}} \rho_{\mathrm{C}} \mathrm{e}^{2 \mathrm{i} \omega T_{\mathrm{A}}}-\rho_{\mathrm{C}} \rho_{\mathrm{S}} v^{2} \mathrm{e}^{\mathrm{i}\left(2 \omega T_{\mathrm{S}}+\delta\right)}+\rho_{\mathrm{R}} \rho_{\mathrm{S}} v^{2} \mathrm{e}^{\mathrm{i}\left[2 \omega\left(T_{\mathrm{A}}+T_{\mathrm{S}}\right)+\delta\right]}},
$$

for signals fed into the arm cavity (setting $b_{2}=0$ ), and 


$$
G_{2}(\omega, \delta)=\frac{a_{0}}{b_{2}}=\frac{\tau_{\mathrm{S}} v \mathrm{e}^{\mathrm{j} \omega T_{\mathrm{S}}}\left(1-\rho_{\mathrm{R}} \rho_{\mathrm{C}} \mathrm{e}^{2 \mathrm{i} \omega T_{\mathrm{A}}}\right)}{1-\rho_{\mathrm{R}} \rho_{\mathrm{C}} \mathrm{e}^{2 \mathrm{i} \omega T_{\mathrm{A}}}-\rho_{\mathrm{C}} \rho_{\mathrm{S}} v^{2} \mathrm{e}^{\mathrm{i}\left(2 \omega T_{\mathrm{S}}+\delta\right)}+\rho_{\mathrm{R}} \rho_{\mathrm{S}} v^{2} \mathrm{e}^{\mathrm{i}\left[2 \omega\left(T_{\mathrm{A}}+T_{\mathrm{S}}\right)+\delta\right]}},
$$

for signals fed into the signal extraction cavity ( $\operatorname{cetting} b_{1}=0$ ). Here $\tau$ and $\rho$ denote the amplitude transmission and reflection coefficients of the respective mirrors. The angular frequency $\omega$ denotes the light frequency offset with respect to the carrier frequency (thus $\omega= \pm \omega_{\mathrm{g}}$ for the upper and lower sideband, respectively). The phase $\delta$ represents the detuning of the signal extraction cavity, with $\delta=0$ for non-detuned (broadband) $\mathrm{RSE}, \delta=\pi$ for signal recycling and all other values representing detuned cases. $T_{\mathrm{S}}=L_{\mathrm{S}} / c$ and $T_{\mathrm{A}}=L_{\mathrm{A}} / c$ are the one-way light travel times in the signal extraction cavity and arm cavity, respectively. The losses of the arm cavity are modeled by a finite transmittance of the rear mirror $0<\tau_{R}^{2} \ll 1$, and the losses in the signal extraction cavity (beam splitter, imperfect contrast, etc.) by an amplitude transmittance $v \lesssim 1$. If the signal is a true gravitational wave acting on the whole length of the cavity, $G_{1}$ must be multiplied by the factor $\sin \left(\omega T_{\mathrm{A}}\right) / \omega T_{\mathrm{A}}$ ( see e.g. Ref. [12]), which can however be neglected for our experiment.

We use external modulation (see e.g. Ref. [13] and references therein) to detect the signal sidebands in our experiment. In the Mach-Zehnder interferometer used for this purpose, the light eventually leaving the Michelson interferometer through $\mathrm{M}_{\mathrm{S}}$, with amplitude $a_{\mathrm{s}}$, is superimposed with a reference beam that has been phase modulated at an angular frequency $\omega_{\mathrm{e}}$. A relative phase shift $\psi$ is introduced between signal and reference beam before they are superimposed in order to adjust for maximum output signal. Synchronous demodulation of the resulting photocurrent at the modulation frequency $\omega_{\mathrm{e}}$ produces an output signal given by

$$
y=y_{0}\left[\operatorname{Re}\left(a_{\mathrm{s}}\right) \sin \psi+\operatorname{Im}\left(a_{\mathrm{s}}\right) \cos \psi\right]=y_{0} \operatorname{Im}\left(a_{\mathrm{s}} \mathrm{e}^{\mathrm{i} \psi}\right),
$$

where all constant factors have been included in $y_{0}$.

The signal wave $a_{\mathrm{s}}$ consists of two sidebands $a_{+}$and $a_{-}$, which are not necessarily symmetric in the detuned cases of RSE (or signal recycling),

$$
a_{\mathrm{s}}=\mathrm{i} a_{+} \mathrm{e}^{\mathrm{i} \omega_{\mathrm{g}} t}+\mathrm{i} a_{-} \mathrm{e}^{-\mathrm{i} \omega_{g} t} .
$$

The factors $\mathrm{i}$ arise from setting the carrier phase to zero. For the output signal as detected by external modulation we get from Eqs. (3) and (4),

$$
y=\operatorname{Re}\left(A \mathrm{e}^{\mathrm{i} \omega_{\mathrm{g}} t}\right), \quad \text { with } A=a_{+} \mathrm{e}^{\mathrm{i} \psi}+a_{-}^{*} \mathrm{e}^{-\mathrm{i} \psi},
$$

where $A$ represents the complex amplitude of the output signal of frequency $\omega_{\mathrm{g}}[6]$. The asterisk denotes complex conjugation.

Thus the transfer function of the interferometer up to the demodulated detector output for phase modulation signals of frequency $\omega_{\mathrm{g}}$ generated inside either the arm cavity or signal extraction cavity is given (neglecting common constant factors) by

$$
\begin{aligned}
& H_{1}\left(\omega_{\mathrm{g}}, \delta, \psi\right)=G_{1}\left(\omega_{\mathrm{g}}, \delta\right) \mathrm{e}^{\mathrm{i} \psi}+G_{1}^{*}\left(-\omega_{\mathrm{g}}, \delta\right) \mathrm{e}^{-\mathrm{i} \psi}, \\
& H_{2}\left(\omega_{\mathrm{g}}, \delta, \psi\right)=G_{2}\left(\omega_{\mathrm{g}}, \delta\right) \mathrm{e}^{\mathrm{i} \psi}+G_{2}^{*}\left(-\omega_{\mathrm{g}}, \delta\right) \mathrm{e}^{-\mathrm{i} \psi},
\end{aligned}
$$

since both sidebands have been produced with the same amplitude by the phase modulation.

Note that the transfer function of the interferometer depends on $\psi$, the relative phase between signal wave and reference wave. This becomes important in detuned cases of RSE (or signal recycling), where both sidebands will generally have different amplitudes. One extreme is the "single sideband" case, where the signal wave $a_{\mathrm{s}}$ is dominated by only one of the two sidebands. This approximately happens near the sensitivity peak of a detuned interferometer. Then the dependence on $\psi$ is small, as can be seen from Eq. (5) by setting $a_{-}=0$.

When the two sidebands have comparable amplitudes, however, the amount of detected output signal depends sinusoidally on $\psi$. It turns out that there is not one fixed optimum detection phase $\psi$ for all signal frequencies 


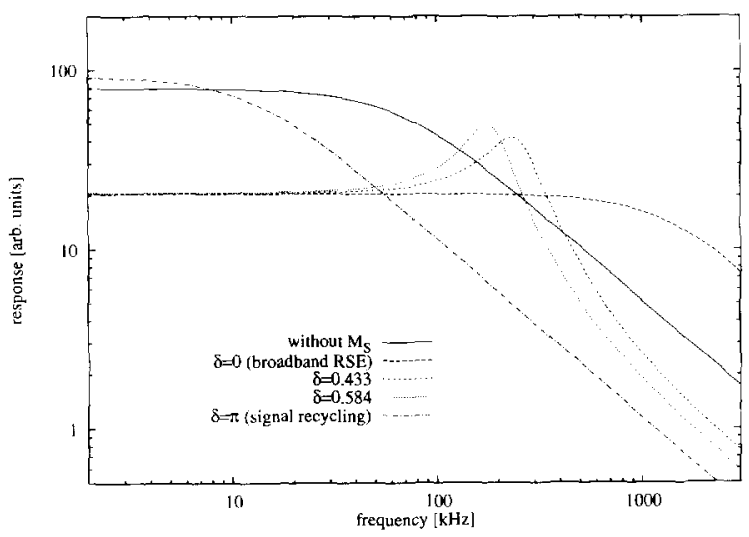

Fig. 3. Calculated frequency response of our table-top interferometer.

simultaneously. The difference between the optimum $\psi$ for low and for high frequencies (in relation to the peak frequency) is roughly $\pi / 2$.

Fig. 3 shows the computed frequency response $\left|H_{1}(\omega, \delta, \psi=0)\right|$ of our table-top interferometer, using the parameters given in the next section. We have plotted the responses for the non-detuned cases of RSE and signal recycling ( $\delta=0$ and $\delta=\pi$, respectively), two detuned operating points used in our experiment, and the response without any mirror $\mathrm{M}_{\mathrm{S}}$ for comparison. For a clear experimental demonstration of an increase in sensitivity we chose the detuned operating points, since test signals were applied by a PZT having a few $100 \mathrm{kHz}$ bandwidth at most. Furthermore, the characteristic peaks in the frequency response allow a better comparison with theory. In order to eliminate the effect of PZT resonances we compared the response with $\mathrm{MS}_{\mathbf{S}}$ locked to its proper position to the response with $\mathbf{M}_{\mathbf{S}}$-removed.

\section{Experiment}

In our experiment (Fig. 4), the interferometer was illuminated by approximately $300 \mathrm{~mW}$ of single-mode light at $514.5 \mathrm{~nm}$ from an INNOVA-90 $\mathrm{Ar}^{+}$Laser through two Faraday isolators (made by Optics for Research). Its frequency can be controlled by an intra-cavity Pockels cell and a slow PZT shifting one mirror. The light is phase modulated at $12 \mathrm{MHz}$ by a Gsänger PM-25 Pockels cell PC1, with a modulation index of approximately $0.25 \mathrm{rad}$. The arm cavities (length $40 \mathrm{~cm}$ ) consist of flat coupling mirrors $\left(\mathrm{M}_{\mathrm{C} 1}\right.$ and $\mathrm{M}_{\mathrm{C} 2}$ ) with $1850 \mathrm{ppm}$ power transmittance (measured average), and curved reflectors $\left(M_{R 1}\right.$ and $\left.M_{R 2}, R=1 \mathrm{~m}\right)$ mounted on massive aluminum cylinders. Total cavity losses (excluding $\tau_{\mathrm{C}}^{2}$ ) were measured as $350 \mathrm{ppm}$ (the main loss originating from the coating of the rear reflecting mirrors), yielding a finesse of approximately 3000 and a power reflectivity of the cavities of about $50 \%$ at resonance. With two mode-matching lenses, more than $90 \%$ of the incoming power was coupled into the cavity's fundamental mode.

Glass plates (BP1 and BP2) mounted almost under the Brewster angle reflect $0.5 \%$ of the light power returning from the cavities to the photodetectors PD1 and PD2. Their photocurrents are demodulated at $12 \mathrm{MHz}$ and used for stabilization of the laser frequency and the second cavity's length, employing the Pound-Drever method [14]. The system was operated in air and thus very sensitive to acoustic disturbances, necessitating high gain and broad bandwidth for those loops. The first loop (with PD1) locks the laser to the first cavity with a unity-gain frequency of approximately $120 \mathrm{kHz}$. The second loop (with PD2) locks the length of the second cavity to the laser by adjusting the cavity's length with one slow high-efficiency and one fast PZT, the latter being mounted on an acoustic delay line to reduce low-frequency mechanical resonances. This loop had a unity gain frequency of approximately $40 \mathrm{kHz}$. 


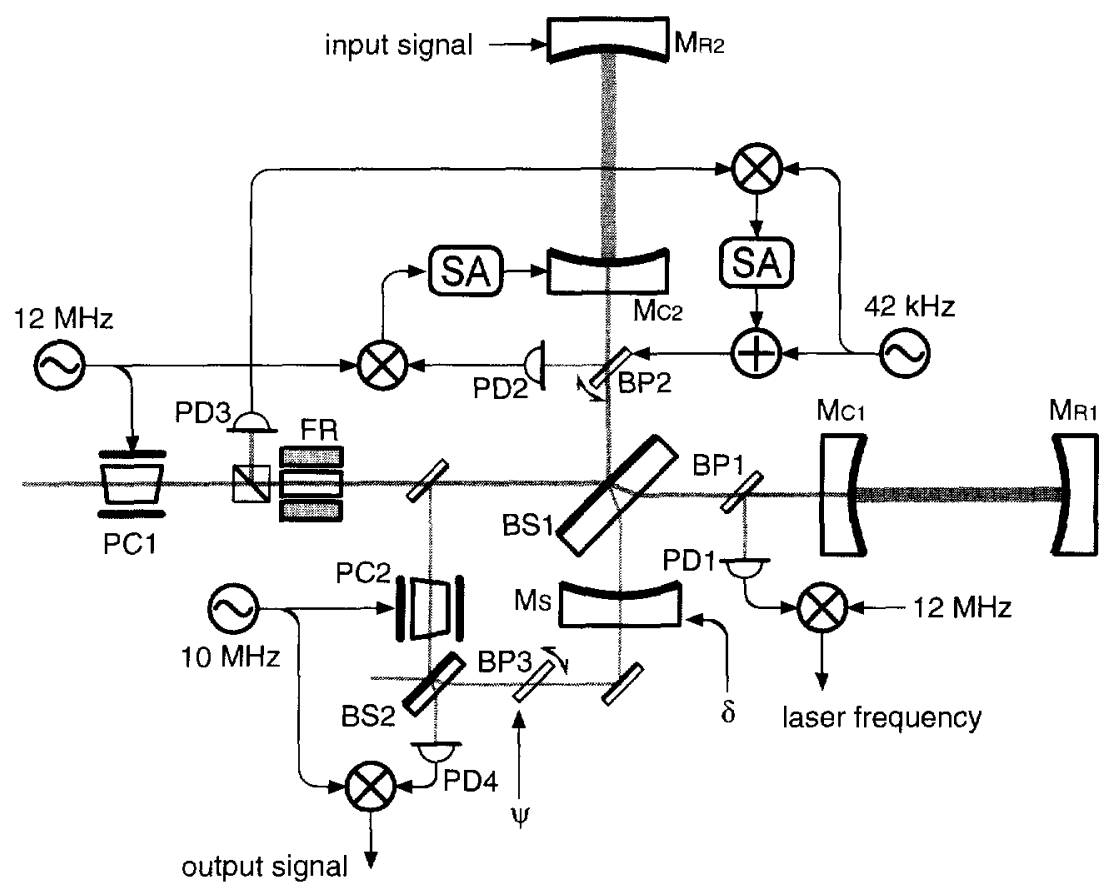

Fig. 4. Simplified diagram of our experimental setup, showing only the control loops for the arm cavities and the Michelson interferometer. FR is a Faraday rotator, PC are Pockels cells, $\Theta, \oplus$ and $\otimes$ electronic oscillators, adders and mixers respectively, BS are beam splitters, BP are glass plates in or near the Brewster angle, PD photodiodes and SA servo amplifiers. See also Fig. 5.

A phase modulation of $42 \mathrm{kHz}$ (internal modulation) was applied between the beam splitter and the second cavity by dithering the Brewster plate BP2, which was mounted on a galvanometer scanner allowing tilting about small angles. A tilt of $0.03^{\circ}$ was sufficient to change the optical path length by $\lambda / 2$, and the modulation index at $42 \mathrm{kHz}$ was approximately $5 \mathrm{mrad}$. The light returning towards the laser was directed onto photodetector PD3 by one of the Faraday isolators. The photocurrent was demodulated at $42 \mathrm{kHz}$ and fed back to BP2 through an appropriate filter in order to lock the Michelson interferometer to a dark fringe at its output. The unity-gain frequency of this loop was approximately $700 \mathrm{~Hz}$. Another purpose of this $42 \mathrm{kHz}$ modulation is to continually adjust the external modulation's phase relationship, as described below. With both arm cavities locked, the interference minimum was approximately $1 \%$ of the power at the maximum.

Test signals up to $500 \mathrm{kHz}$ were fed into the interferometer at the fast PZT holding $\mathrm{M}_{\mathrm{R} 2}$. This phase modulation in only one arm can be separated into two components of equal magnitude, a common mode component directed towards the laser, and a differential component, which represents the signal of interest. The reference beam for the external modulation was taken from the light returning towards the laser and was phase modulated at $10 \mathrm{MHz}\left(=\omega_{\mathrm{e}} / 2 \pi\right)$ by another PM-25 Pockels cell (PC2 in Fig. 4). The relative phase $\psi$ between signal and reference beam was introduced via another galvanometer-tilted Brewster plate BP3. After synchronous demodulation (at $10 \mathrm{MHz}$ ), the photocurrent of photodetector PD4 represents the interferometer's output signal.

The phase $\psi$ was controlled so as to maximize the amplitude of the $42 \mathrm{kHz}$ component in the output signal originating from the phase modulation at BP2. The amplitude at $42 \mathrm{kHz}$ was detected by synchronous demodulation of the interferometer output at $42 \mathrm{kHz}$. This amplitude was maximized by dithering $\psi$ with 260 $\mathrm{Hz}$, demodulating the $42 \mathrm{kHz}$ amplitude at $260 \mathrm{~Hz}$ and feeding back the resulting error signal to BP3 through an appropriate loop filter. This loop is not shown in Figs. 4 and 5. 


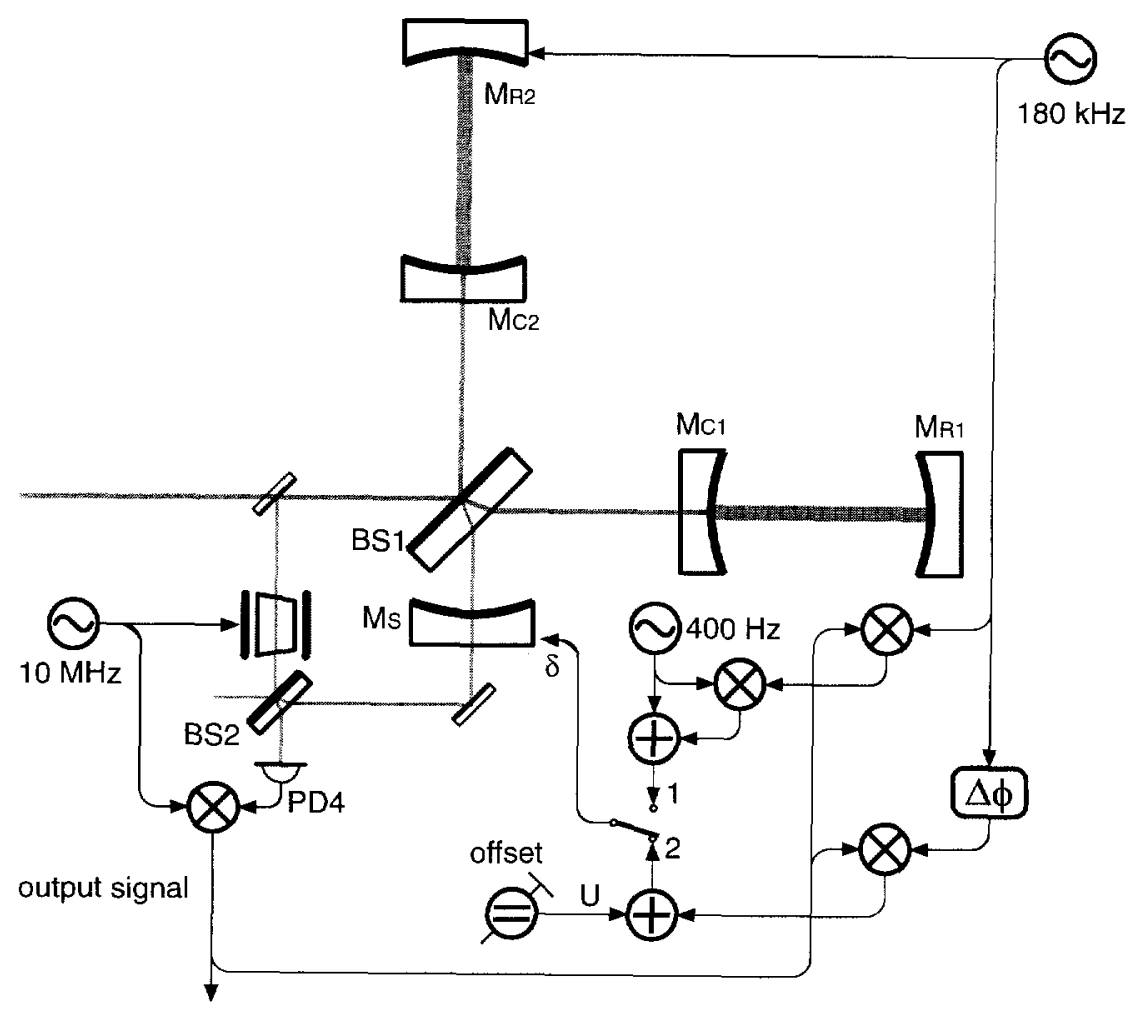

Fig. 5. Control loops for the signal extraction mirror $M_{S}$. In addition to the symbols used in Fig. 4, $\Delta \phi$ represents an electronic phase shifter and $\ominus$ an adjustable DC voltage.

The curved signal extraction mirror $\mathrm{M}_{\mathrm{S}}(R=1 \mathrm{~m})$ had a power reflectivity of $82 \%$ and was placed $40 \mathrm{~cm}$ away from the flat mirrors $\mathrm{M}_{\mathrm{Cl}}$ and $\mathrm{M}_{\mathrm{C} 2}$, so that its curvature matched the wavefront curvature. The amplitude loss factor of the signal extraction cavity, $v$ in Eqs. (1) and (2), was estimated as $\sqrt{1-0.005}$, taking only the losses by BP1 and BP2 into account. The influence of this factor on the frequency response is small.

Two different schemes to lock the position of $\mathrm{M}_{\mathrm{S}}$ (using another PZT) were investigated. Both schemes use a $180 \mathrm{kHz}$ calibration signal (with an amplitude corresponding to less than $1 \mathrm{pm}$ movement), which was also fed to $\mathrm{M}_{\mathrm{R} 2}$ (see Fig. 5).

The first scheme (switch position 1 in Fig. 5) was aiming at a peak sensitivity at $180 \mathrm{kHz}$. The amplitude of the $180 \mathrm{kHz}$ calibration signal at the output was detected by synchronous demodulation. In a scheme similar to the control of $\psi$ described above, the position of $\mathrm{M}_{\mathrm{S}}$ was dithered with $400 \mathrm{~Hz}$. The $180 \mathrm{kHz}$ amplitude was again synchronously demodulated at $400 \mathrm{~Hz}$ to obtain an error signal, which was fed back to $\mathrm{M}_{\mathrm{S}}$. For real gravitational wave detectors, this scheme will not be the best choice, since one presumably will not want to introduce test signals just at the sensitivity peak, and because the frequency of that peak needs to be dithered to obtain an error signal.

The scheme was, however, useful to acquire lock of the different coupled loops and to adjust the electronics for the second scheme (switch position 2 in Fig. 5). Here $M_{S}$ is no longer dithered, but instead controlled in such a way as to maintain the amplitude of the $180 \mathrm{kHz}$ signal at the output at a predefined constant level $U$, similar to a concept initially proposed by Strain for the use with signal recycling [15]. The $180 \mathrm{kHz}$ amplitude is again obtained by synchronous demodulation, now, however, with a different and adjustable demodulation phase $\Delta \phi$. If the level $U$ is chosen somewhere between the extreme amplitude values, the error signal obtained 


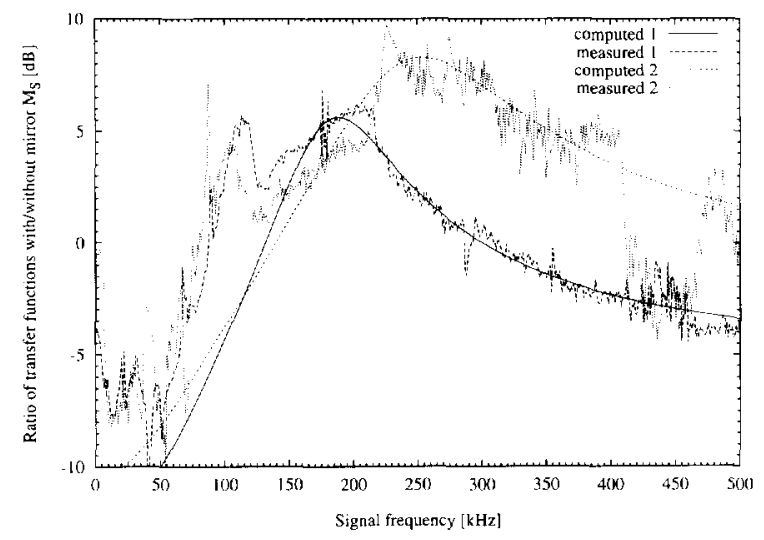

Fig. 6. Measured and computed ratio of transfer functions with and without signal extraction mirror $\mathrm{M}_{\mathrm{S}}$ for the two schemes of controlling Ms.

by subtracting the instantaneous amplitude from $U$ has the proper sign on both sides of the desired operating point. There are two degrees of freedom $(U$ and $\Delta \phi$ ) which allow to select the desired tuning $\delta$ (and hence the detector's frequency response) in a wide range.

In order to measure the transfer function, test signals from $0-500 \mathrm{kHz}$ (with amplitudes corresponding to mirror movements of less than $1 \mathrm{pm}$ ) were fed to $\mathrm{M}_{\mathrm{R} 2}$. The magnitude of the output signal was simultaneously monitored at the signal frequency using a Marconi $2380 / 2382$ spectrum analyzer, which was also used to generate the swept sine test signals. To observe the effect of the signal extraction mirror $\mathbf{M}_{S}$, each measurement was repeated with $\mathrm{M}_{S}$ removed. The ratio of these two transfer functions was compared with the theoretically expected ratio

$$
M(\omega, \delta)=\frac{H_{1}\left(\omega, \delta, \psi_{42}(\delta)\right)}{H_{1}^{0}(\omega, \psi \equiv 0)}
$$

Here $H_{1}^{0}(\omega, \psi \equiv 0)$ is obtained from $H_{1}$ by setting $\rho_{\mathrm{S}}=0$, and $\psi_{42}(\delta)$ is described below.

In the interpretation of our data, special attention must be paid to the phase $\psi$ of the Mach-Zehnder interferometer, since the interferometer's transfer function depends on $\psi$, as described in Section 2 . Without $\mathrm{M}_{\mathrm{S}}$, the $42 \mathrm{kHz}$ signal used to adjust $\psi$ arrives at the output with a certain phase which we take as zero. The 42 $\mathrm{kHz}$ demodulator's reference phase was adjusted for this case and not changed afterwards. With $\mathrm{M}_{\mathrm{S}}$ in place, however, the $42 \mathrm{kHz}$ signal experiences a change in magnitude and phase according to Eq. (7). In our setup, the control loop for $\psi$ continually maximizes the $42 \mathrm{kHz}$ amplitude as determined by demodulation with the preset phase. This corresponds to maximizing $\operatorname{Re}\left[H_{2}(2 \pi \times 42 \mathrm{kHz}, \delta, \psi)\right]$. We define the phase $\psi_{42}(\delta)$ by this maximum condition, in accordance with the $\psi$ that appears in the experimental situation. It was computed numerically and was found to vary between -0.6 and $+0.6 \mathrm{rad}$, depending on $\delta$.

\section{Results and conclusion}

Fig. 6 shows the measured results. The ratios of the interferometer's measured transfer functions with and without mirror $\mathrm{M}_{\mathbf{S}}$ are shown for the two different schemes of controlling $\mathrm{M}_{\mathbf{S}}$. The computed ratios are shown for comparison.

The curves labelled 1 refer to the first scheme (setting the peak sensitivity to $180 \mathrm{kHz}$ by dithering $\mathrm{M}_{\mathrm{S}}$ ). The tuning $\delta$ was $0.584 \mathrm{rad}$ (found by a nonlinear fit), and the corresponding Mach-Zehnder phase was 
$\psi=\psi_{42}(\delta)=-0.336 \mathrm{rad}$. At frequencies around and above the peak, theory and experiment agree reasonably well.

The discrepancies at lower frequencies were found to result from an interaction with the Pound-Drever systems used to keep the two arm cavities resonant. With $\mathrm{M}_{S}$ in place, the two cavities are no longer independent. The laser frequency is stabilized to the first cavity with a unity-gain frequency of approximately $120 \mathrm{kHz}$ (without $\mathrm{M}_{\mathrm{S}}$ ). The gain of this loop is modified by $\mathrm{M}_{\mathrm{S}}$, because the detuned signal extraction cavity causes additional optical feedback and introduces extra phase shifts in the loop. This was verified by changing the overall loop gain of the frequency stabilization and observing a shift in frequency and height of the $120 \mathrm{kHz}$ peak in Fig. 6.

Another effect is caused by the asymmetry between the two Pound-Drever systems. As described in Section 3, the second arm cavity is kept resonant with a unity-gain frequency of only a few $10 \mathrm{kHz}$, considerably lower than the $120 \mathrm{kHz}$ of the first cavity loop. The test signal being fed into the second cavity appears, after reflection by $\mathrm{M}_{\mathbf{S}}$, as a frequency deviation on photodetectors PD1 and PD2. Here it is amplified and fed back to the laser frequency and the length of the second cavity, respectively. At those frequencies where some loop gain remains, the theory described in Section 2 is no longer valid, especially when the gains for the two arms are not identical.

Both these effects arise from our specific experimental setup and will not appear in the planned large-scale detectors if the laser frequency is stabilized to either an independent reference or to the power-recycling cavity instead of one arm cavity, and if the unity-gain frequency of the loops used to lock the arm cavities is below the observation bandwidth.

The curves labelled 2 were obtained by locking $\mathrm{M}_{\mathrm{S}}$ with the second scheme (maintaining the $180 \mathrm{kHz}$ output signal at a constant level). A sensitivity peak at approximately $250 \mathrm{kHz}$ was obtained, corresponding to $\delta=0.433 \mathrm{rad}$. A constant of $1.8 \mathrm{~dB}$ had to be added to the theoretical curve for a best fit, which can be understood by the misalignment of the Mach-Zehnder interferometer introduced by removing $\mathrm{M}_{\mathrm{S}}$ and the lens used to compensate the lensing effect of the curved mirror substrate. Around $420 \mathrm{kHz}$ the PZT response was so small that both signals were buried in the noise. In further experiments (not shown), the sensitivity peak could be shifted to as high as $400 \mathrm{kHz}$, more than twice the calibration signal frequency.

\section{Conclusion}

We have, for the first time, operated a resonant sideband extraction interferometer at a detuned operating point. The frequency response agrees well with theory. A coupling of formerly independent loops by optical feedback was observed, and requires further investigation. Another interesting point is the detection of the signal sidebands by external modulation in a Mach-Zehnder type interferometer. We have confirmed experimentally the validity of the expressions describing the output in the general (detuned) case, Eqs. (3)-(7). In the detuned case of signal extraction or signal recycling, the phase of this Mach-Zehnder interferometer cannot be optimized for all signal frequencies simultaneously.

\section{References}

[1] A. Abramovici et al., Science 256 (1992) 325.

[2] C. Bradaschia et al., Nucl. Instr. Meth. Phys. Res. A 298 (1990) 518.

[3] K. Danzmann et al., in: Proc. first Edoardo Amaldi Conf. on Gravitational wave experiments, Frascati 1994 (World Scientific, Singapore, 1995).

[4] B.J. Meers, Phys. Rev. D 38 (1988) 2317.

[5] P.R. Saulson, Fundamentals of interferometric gravitational wave detectors (World Scientific, Singapore, 1994).

[6] J. Mizuno, Ph.D. Thesis, Universität Hannover and Max-Planck-Institut für Quantenoptik, Garching (1995); also available as MPQReport 203, July 1995. 
[7] W. Winkler et al., Phys. Rev. A 44 (1991) 7022.

[8] W. Winkler et al., Opt. Commun. 112 (1994) 245.

[9] K.A. Strain et al., Phys. Lett. A 194 (1994) 124.

[10] J. Mizuno et al., Phys. Lett. A 175 (1993) 273.

[11] B.J. Meers, Phys. Lett. A 142 (1989) 465.

[12] J.A. Lobo, Class. Quantum Grav. 9 (1992) 1385.

[13] C.N. Man et al., Phys. Lett. A 148 (1990) 8.

[14] R.W.P. Drever et al., Appl. Phys. B 31 (1983) 97.

[15] K.A. Strain, private communication (1992). 